\title{
Constructing a database for low cost identification of Gram negative rods in clinical laboratories
}

\author{
P CLAYTON, RKA FELTHAM, CJ MITCHELL, PHA SNEATH* \\ From the Public Health Laboratory, Leicester Royal Infirmary, Leicester, and the *Department of \\ Microbiology, University of Leicester, Leicester
}

SUMMARY A database was constructed for the routine identification of Enterobacteriaceae an Gram negative rods growing aerobically on MacConkey agar. The test methods were basedi primarily on multipoint inoculation technology. The final database was constructed from the laboratory results of 4989 clinical isolates and 66 reference strains and was extensively analysegु and evaluated. The proposed scheme is rapid, reliable, and cheap.

Routine methods for identifying Gram negative bacilli growing on MacConkey agar vary between laboratories. Many laboratories will identify isolates from serious clinical infections, such as those from blood cultures and cerebrospinal fluids, but provide only minimal identification of organisms from specimens such as urine. One of the major factors in this differentiation between specimens, apart from the clinical or epidemiological importance of the identification, is cost.

The methods used to identify Gram negative rods growing aerobically on MacConkey agar range from conventional tests ${ }^{1}$ to kits such as API20E. ${ }^{2} \mathrm{~A}$ set of conventional tests is difficult to standardise, time consuming - and therefore expensive to prepareand may take up to five days to produce a definitive result. Once results are recorded they must be subjectively compared with diagnostic tables. ${ }^{13} \mathrm{~A}$ few laboratories have introduced a computer assisted form of identification. ${ }^{4}$

Identification kits are standardised, usually take 24 to 48 hours for interpretation of results, and provide either a full computer assisted service with microcomputer or a printout of the most common result patterns, or profiles. They are, however, expensive to purchase and for this reason tend to be used only for highly important isolates.

We report here a simple system for the identification of Enterobacteriaceae and other Gram negative rods that grow aerobically on MacConkey agar. The system comprises conventional tests, using substrates incorporated into agar plates and a multi-

Accepted for publication 6 February 1986

point inoculation system similar to that used for the antibiotic susceptibility testing breakpoint method. This system provides an identification in 24 hour $\vec{\varepsilon}$ makes use of computer assisted technology-eithe्क with an interactive terminal or a profile printout and is simple and cheap to perform.

\section{Material and methods}

MEDIA AND QUALITY CONTROL

Unless otherwise stated, all tests were performe $\vec{\not}$ using a 21 pin multipoint inoculator on substrate incorporated agar plates. Three of the pin positions were used for internal quality control of all batches: The organisms used were laboratory isolates $\mathrm{BP}$ (Escherichia coli), BP8 (Proteus mirabilis), and BPO (Pseudomonas aeruginosa). All tests were incubated. at $37^{\circ} \mathrm{C}$ for 18 to 24 hours. Motility was also tested room temperature.

Oxidase was tested by smearing the test organism of to filter paper moistened with a $1 \%(\mathrm{v} / \mathrm{v})$ aqueous solution of tetramethyl-para-phenylenediamine dihtg. drochloride.

Phenylalanine deamination was tested using Mas (Mast Laboratories Ltd, Bootle, Merseysided England) phenylalanine agar (IDM 31). A browmy black coloured colony indicated a positive result. ${ }^{\omega}$ Gelatin hydrolysis was tested using Mast gelatin agă (IDM 24). After incubation the colonies were sepa: rated from each other using a scalpel and then the plates were flooded with acidic mercuric chloride Clear areas around the colonies indicated positive gelatinase production.

The indole test was performed in $5 \mathrm{ml}$ aliquots of peep tone water ${ }^{1}$ in bijoux bottles. A few drops of Kovacs? indole reagent were added after incubation, and a red 
colour in the reagent layer indicated a positive result. Urease production was tested using Mast urea agar base (Multipoint) (IDM 32). A positive reaction was indicated by a purple halo and colony on an orangeyellow plate.

Hydrogen sulphide production was tested using Mast hydrogen sulphide agar (IDM 25). Positive organisms showed as black colonies.

Acid from glucose, mannitol, rhamnose, sucrose, and inositol were tested using Mast carbohydrate peptone agars (IDM 3, 6, 8, 10, and 4). The indicator was bromo-thymol blue, and a positive fermentation reaction was indicated by a yellow halo round the colony on a blue plate.

Citrate utilisation was tested using Mast citrate agar (IDM 23). This was a modification of Simmons' method, and organisms able to use citrate as their sole carbon source both grew and changed the medium from green to blue.

ONPG production was tested using Mast ONPG agar base (IDM 29). After multipoint inoculation each suspension drop was separated using a sterile scalpel blade to cut thin channels between the inocula. ${ }^{7}$ This prevented cross colouration and facilitated the interpretation of a pale yellow positive colour.

Lysine decarboxylase production was tested using Mast LDC agar (IDM 26). This was a modification of Mancani's aerobic technique, ${ }^{8}$ and positive results were shown as a purple colour; negatives showed as yellow.

Malonate utilisation was tested using Mast malonate agar (IDM 27). A positive result was indicated by a blue colour from a green plate.

Motility was tested both at $37^{\circ} \mathrm{C}$ and room temperature using Mast motility test agar (IDM 28), which contained the dye triphenyltetrazolium chloride (TTC). The agar was dispensed as $2 \mathrm{ml}$ quantities in each of the 25 chambers in a compartmentalised Petri dish. The test organisms were stabbed into each chamber with a straight wire, and after overnight incubation positive isolates gave a diffuse pink cloud throughout the chamber for either one of the incubation temperatures. Negative isolates gave a red inoculation track for both temperatures.

An extra test (Oxoid CN agar CM559 plus SR 102 supplement) was used as a confirmatory test for Pseudomonas spp. This was not included in the principal database but used only as confirmation on occasions.

\section{COMPARATIVE COSTING}

A comparative costing exercise was carried out between the methods detailed here and a commercial kit (API20E, API Laboratories, Basingstoke, Hampshire).

A set of 18 organisms, chosen at random from routine clinical material, was tested with the multipoint
Table 1 Comparative costing: multipoint system $v$ API2OE

\begin{tabular}{llr}
\hline Item/18 organisms & $\begin{array}{l}\text { Multipoint } \\
\text { system } \\
(£)\end{array}$ & API20E \\
\hline Medium cost & 1.59 & $(f)$ \\
Preparation & 0.845 & 23.04 \\
Set up labour & 1.94 & 0.00 \\
Reading labour & 1.745 & 3.72 \\
Total for 18 organisms & 6.12 & 2.92 \\
Total for 1 organism & $0.34^{*}$ & 29.68 \\
\hline
\end{tabular}

*This value includes three quality control strains per set of 18 organisms.

system and through API20E kits. Table 1 shows the costs of materials, labour for media preparation, labour for setting up tests and for reading and interpretation. The costs of the media were for price lists dated September 1985. All timings were performed for each test method by two medical laboratory scientific officers. The results shown in Table 1 are the averages of these two sets of figures. The mid point of the 1984 Whitley council salary scale was used and gave a value of $£ 0.065$ per medical laboratory scientific officer's minute.

\section{DATABASE CONSTRUCTION}

This was carried out in two stages, a preliminary database being used to provide a skeleton profile list from which a full list was finally prepared.

The initial database was constructed using data for 56 tests from Bascomb, Lapage, Willcox, and Curtis. ${ }^{9}$ The computer analysis programs CHARSEP ${ }^{10}$ and DIACHAR $^{11}$ were used to define the most useful 16 of these tests; these were extracted from the full database into a subset. The quality of this smaller database was tested using the programs OVERMAT ${ }^{12}$ and MOSTTYP, ${ }^{13}$ as used by Feltham and Sneath ${ }^{14}$ for Gram positive aerobic cocci. Some of the bacterial groups were amalgamated when they could not be adequately differentiated with the chosen tests. This preliminary database was used to generate a set of octal profiles using the computer programs PROFGEN, PROFSORT, and PROFPRINT.

The program PROFGEN generated a list of the most likely six digit profiles with corresponding taxon name for each taxon within the matrix. The program

Table 2 Sample of full profile list

\begin{tabular}{lll}
\hline Profile & Taxon name & Probability \\
\hline 017404 & Citrobacter freundii & 0.961581 \\
& Salmonella typhi & 0.015363 \\
017410 & Klebsiella rhinoscleromatis & 0.990932 \\
017440 & Citrobacter freundii & 0.930282 \\
& Klebsiella ozaenae & 0.032819 \\
017444 & Citrobacter freundii & 0.995975 \\
\hline
\end{tabular}


PROFSORT used the data produced by PROFGEN to print a numerical list of profiles with the taxon name beside each profile. Specified taxon names also had asterisks printed alongside their names to denote that serology would be a useful extra diagnostic test. If any duplicate profiles were produced the output from PROFPRINT was used as reference.

The program PROFPRINT, using the output from PROFGEN, gave a computer assisted identification against the matrix for each listed profile. The output from this program printed the profile, the single highest taxon name, and probability of identification by the Willcox probability coefficient, ${ }^{4}$ provided the highest probability was at least 0.97 . If the highest value was less than 0.6 then this was regarded as unidentified; for all other values between 0.61 and 0.96 the profile, top two taxon names and their corresponding probability scores were printed (Table 2 ). This database was evaluated using the following procedures. A total of 5055 strains were tested against this database using the substrate incorporation methods described above. Sixty six reference strains from the Leicester clinical culture collection were used in conjunction with 4989 fresh clinical isolates. If any isolate did not identfy according to the laboratory protocol they were further identified using API20E. The multipoint methods were repeated, and if consistent, the "new" profile was incorporated into the list.

The profiles from the 5055 strains were analysed by the computer program PROFMAT to generate a new database, wholly based on the actual results obtained from the multipoint methods in our laboratory. The data for the program comprised a list of bacterial taxon names, their corresponding profiles, together with the number of times each profile for that taxon had occurred. The program carried out the analysis in three stages: sort all bacterial names together with corresponding profile data; calculate sequential

Table 3 Improved Enterobacteriaceae plus related organisms database $(B)$

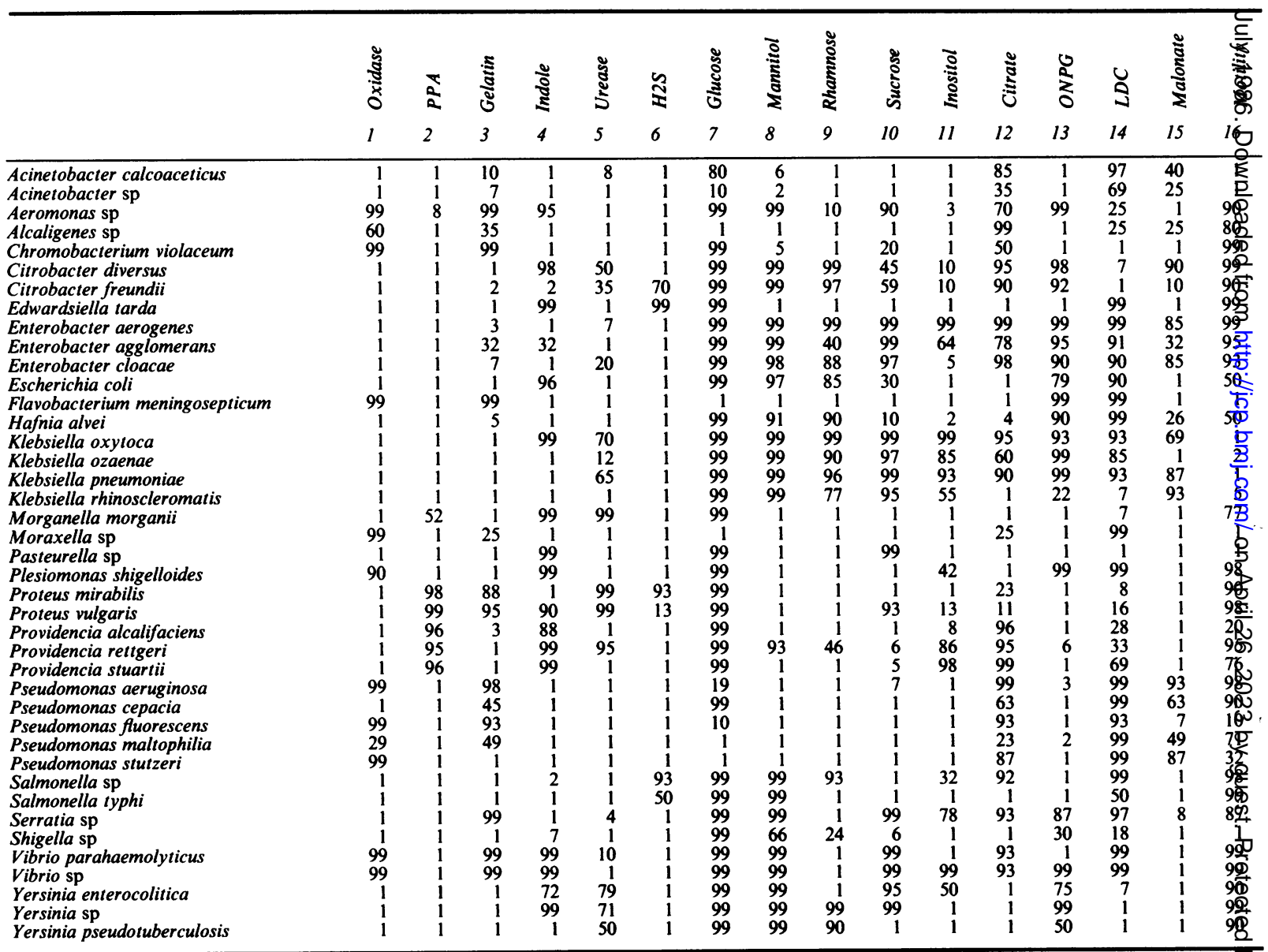

PPA = Phenylalanine; H2S = hydrogen sulphide; LDC = lysine decarboxylase production 
summated results for each taxon; construct and print probabilities of positive results for each taxon. This database was analysed using the OVERMAT and MOSTTYP computer programs, and some species were again amalgamated to ensure a good identity (identification level of 0.95) to at least genus level.

The test order was changed after studying the conclusions obtained from the database analysis programs CHARSEP and DIACHAR. Ease of interpretation was also considered. A list of likely profiles and the bacterial taxon names was produced using PROFGEN and PROFSORT for routine use. A full list of probabilities for identification, together with likely taxa, was produced using PROFPRINT.

\section{DATABASE EVALUATION}

Two methods were used to evaluate the preliminary database A. These were repeated for the improved database B. Evaluation I was based on 100 organisms selected from a set used for evaluating the Autobac IDX. ${ }^{15}$ Each organism had been identified using the Autobac and also API20E. Organisms were identified against the preliminary profile index for database $A$, either to "species" or "genus" level. For database B, the organisms were compared with the full probability profile index generated from PROFPRINT and then against a fuller list of possible profiles generated by PROFGEN or PROFSORT, with $98 \%$ or more being a positive result and $2 \%$ or less, negative.

Evaluation II was much more stringent and entailed four separate sets of 50 organisms chosen from a range of previously identified collection organisms. Each set contained single, duplicate, or triplicate cultures selected and coded at random. The same two methods for identification (Evaluation I and databases $\mathbf{A}$ and $B$ ) were used.

\section{Results}

Table 2 gives a sample of the full profile list. Table 3 shows the improved database, and Table 4 shows the results from the evaluations compared with those of the improved database. Using 59 random paired strains from Evaluation II, there were 22 test differences. For the 16 tests this gave a test error $(p=1 \cdot 17915 \%)$. The 22 test differences comprised five for the motility test and four for the malonate test.

\section{Discussion}

Pease ${ }^{16}$ described a rapid and economic scheme using a multipoint identification system. Only 12 tests were used to distinguish between a selection of 10 genera from the Enterobacteriaceae. The scheme proved reliable, flexible, and rapid in use. Problems were found with diffusion of colour through some of the
Table 4 Percentage identification rates for two evaluations

\begin{tabular}{lll}
\hline No of strains & $\begin{array}{l}\text { Identification } \\
98 \% / 2 \% \text { index }\end{array}$ & $\begin{array}{l}\text { Identification } \\
\text { full index }\end{array}$ \\
\hline I 100 & 96.9 & 94.9 \\
II 200 & 98.5 & 91.8 \\
\hline
\end{tabular}

media, which were solved by anaerobic incubation and an increase in agar concentration. We experienced difficulty with the ONPG and gelatin reactions until each inoculum was segregated using dividing channels. The lysine decarboxylase gave unusual, though consistent results. Mancani ${ }^{8}$ found that inoculation with the prongs just below the agar surface gave stronger reactions. Our motility test proved extremely difficult to interpret using the standard protocol. This test was placed at the end of the list so that any reading errors would limit the likelihood of a misidentification. We found that an extra test for confirmation of Pseudomonas strains was useful but not essential.

Although the profile register contains many profiles where the second identity has a low probability, routine isolates seldom gave such double identities.

The importance of reproducibility has recently been reiterated, and a interlaboratory probability of errors of $6.1 \%$ for the API20E system was found. ${ }^{17}$ The test organisms were "difficult" strains, which had been sent to the National Collection of Type Cultures computer laboratory for further identification. This value was high, however, despite extreme standardisation of production, inoculation, and interpretation using a colour chart. Other authors have shown an intralaboratory reproducibility value of $2 \% .{ }^{18}$

Conventional tests are prone to test errors with an average $6-10 \%{ }^{19}$ between laboratories and values of $2-4 \%$ (on average) within a laboratory. ${ }^{4}$ Our scheme has shown a high degree of intralaboratory reproducibility, but until another laboratory evaluates the method we are unable to comment on interlaboratory figures. The principal reason for test error has been subjective interpretation, which should be solved with training.

The cost and time taken to perform the tests was very attractive when compared with that of a leading commercial kit. The five fold decrease in direct costs and the almost halving of time required for setting up and reading were impressive. The inclusion in our system of three quality control strains per set of 18 organisms could be changed, so that all 21 positions were used for "unknown" strains. The use of these quality control strains permits inbuilt control of media batches and should be used if possible. Higher numbers of organisms may be inoculated per plate, for example, using 36 pins. Either of these options would make the system even more economical, 
although the 36 pin option could present cross contamination problems.

The multipoint system for identification may be used in association with a multipoint antibiotic breakpoint system and becomes yet more cost effective as an integrated laboratory culture and sensitivity system.

A better degree of differentiation would be achieved for more taxa with more tests. The addition of two more tests, after due consideration, together with improvements in the present choice would necessitate reconstructing the database. This should not prove too difficult if records are kept for all the routine identifications and the procedures described in this paper are followed. We are currently investigating a similar scheme for Gram and catalase positive cocci.

We thank Michaela Foster, AM Folwell, C Geary, GT Parish, and SM Hardy for their help in producing the data for this work.

\section{References}

${ }^{1}$ Cowan ST, Steel KG. Manual for the identification of medical bacteria, Second ed. Cambridge: University Press, 1974.

${ }^{2}$ Holmes B, Willcox WR, Lapage SP. Identification of Enterobacteriaceae by the API20E system. J Clin Pathol 1978;31:22-30.

${ }^{3}$ Lennette EH, Balows A, Hausler WJ, Shadomy HJ, eds. Manual of clinical microbiology, 4th ed. Washington, D.C: American Society for Microbiology, 1985.

${ }^{4}$ Lapage SP, Bascomb S, Willcox WR, Curtis MA. Identification of bacteria by computer: general aspects and perspectives. $J$ Gen Microbiol 1973;77:273-90.

${ }^{5}$ Waterworth P. Sensitivity testing by the break point method. Antimicrob Agents Chemother 1981;7:117-26.

${ }^{6}$ Clayton P. An evaluation of breakpoint susceptibility test methods.
Mast Matters 1984;5:10-17.

${ }^{7}$ Buckwold FJ, Ronald AR, Harding GKM. Biotyping of Escherichia coli by a simple multiple-inoculation agar plate technique. J Clin Microbiol 1979;10:275-8.

${ }^{8}$ Mancani JE. Aerobically incubated medium for decarboxylase testos ing of Enterobacteriaceae by replica-plating methods. $\mathrm{J} \mathrm{Clinz}$ Microbiol 1979;10:940-2.

${ }^{9}$ Bascomb S, Lapage SP, Willcox WR, Curtis MA. Identification o을. bacteria by computer: identification of reference strains. $J$ Geff Microbiol 1977;77:291-315.

${ }^{10}$ Sneath PHA. BASIC program for character separation indices from an identification matrix of percent positive characterses Computers and Geosciences 1979;5:349-57.

${ }^{11}$ Sneath PHA. BASIC program for the most diagnostic propertie $\vec{P}$ of groups from an identification matrix of percent positive char $\overrightarrow{-}$ acters. Computers and Geosciences 1980;6:21-6.

12 Sneath PHA. BASIC program for determining overlap between groups in an identification matrix of percent positive characters Computers and Geosciences 1980;6:267-78.

${ }^{13}$ Sneath PHA. BASIC program for determining the besi identification scores possible from the most typical examples when compared with an identification matrix of percent positive् characters. Computers and Geosciences 1980;6:27-34.

${ }^{14}$ Feltham RKA, Sneath PHA. Construction of matrices fo? computer-assisted identification of aerobic Gram-positive, cocci. J Gen Microbiol 1982;128:713-20.

${ }^{15}$ Stevens M, Feltham RKA, Schneider F, Grasmick C, Schaak P Roos P. A collaborative evaluation of a rapid, automated, bacterial identification system: the Autobac IDX. Eur J Clito Microbiol 1984;3:419-23.

${ }^{16}$ Pease A. Biochemical identification of the Enterobacteriaceae using a multipoint inoculation system. Med Lab Sci 1983;400 349-53.

${ }^{17}$ Holmes B, Dawson CA. Misuse and interlaboratory test reproducibility of API 20E system. J Clin Pathol 1985;380 937-41.

${ }^{18}$ Butler DA, Lobregat CM, Gavan TL. Reproducibility of th Analytab (API 20E system). J Clin Microbiol 1975;2:322-6.

${ }^{19}$ Sneath PHA. Test reproducibility in relation to identification Int J System Bacteriol 1974;24:508-23.

Requests for reprints to: Mr P Clayton, Acting Hea MLSO, Public Health Laboratory, Leicester Royad. Infirmary, Infirmary Square, Leicester LE1 5WW, England 